Comida local (hezha), significados, restaurantes, zonas étnicas, China

\title{
LA COMIDA LOCAL Y SU SIGNIFICADO EN LA CHINA CONTEMPORÁNEA: EL CASO DEL SUROESTE DE HUBEI
}

\author{
XU WU \\ Universidad Normal del Este de China
}

El tema de la comida local ha sido ampliamente discutido por los estudiosos desde las perspectivas antropológica, ${ }^{1}$ de la conservación ecológica, ${ }^{2}$ la etnobotánica, ${ }^{3}$ los estudios sobre las mujeres $^{4}$ y la historia política. ${ }^{5} \mathrm{La}$ antropología ha estado interesada en el significado y la comercialización de la comida local en las

Este artículo fue recibido por la dirección de la revista el 17 de abril de 2013 y aceptado para su publicación el 30 de mayo de 2013.

${ }^{1}$ Véase Elizabeth Finnis (ed.), Reimagining Marginalized Foods: Global Processes, Local Places, Tucson, University of Arizona Press, 2012; Nina Etkin, Foods of Association, Tucson, University of Arizona Press, 2009; Nina Etkin (ed.), Eating on the Wild Side: The Pharmacological, Ecological, and Social Implications of using Non-cultigens, Tucson, University of Arizona Press, 1994; Milton Freeman, "Why Mattak and Other Kalaalimerngit [local foods] Matter", en Birgitte Jacobsen (ed.), Cultural and Social Research in Greenland, 95/96: Essays in Honour of Robert Petersen, Nuuk, Ilisimatusarfik-Atuakkiorfik, 1996, pp. 45-53; Richard Wilk, “'Real Belizean Food': Building Local Identity in the Transnational Caribbean”, American Anthropologist, vol. 101, núm. 2, 1999, pp. 244-255.

${ }^{2}$ Gary Paul Nabhan, Where our Food comes From, Washington, Island Press, 2009; Gary Paul Nabhan (ed.), Renewing America's Food Traditions: Saving and Savoring the Continent's most Endangered Foods, White River Junction, Chelsea Green Publishing, 2008; Gary Paul Nabhan, Coming Home to Eat: The Pleasures and Politics of Local Foods, Tucson, University of Arizona Press, 2001.

${ }^{3}$ Manuel Pardo-de-Santayana, Andrea Pieroni y Rajindra K. Puri (eds.), Ethnobotany in the New Europe: People, Health and Wild Plant Resources, Nueva York, Berghahn, 2010; Michael J. Balick y Paul A. Cox, Plants, People and Culture, Nueva York, Scientific American Library, 1996.

${ }^{4}$ Patricia L. Howard (ed.), Women and Plants: Gender Relations in Biodiversity Management and Conservation, Londres-Nueva York, Zed Books, 2003.

${ }^{5}$ James Scott, The Art of not being governed: Anarchist History of Upland Southeast Asia, New Haven-Londres, Yale University Press, 2009; Roland Bergman, "Subsistence Agriculture in Latin America”, en John Super y Thomas Wright (eds.), Food, Politics and Society in Latin America, Lincoln, University of Nebraska Press, 1985, pp. 106-132. 
zonas marginales en su dimensión de alimentos marginales o alimentos étnicos. ${ }^{6}$ En este trabajo se discuten los significados y la comercialización de la comida local propia de las poblaciones más marginadas de las minorías étnicas en China.

La comida local en las zonas étnicas es significativa y multifuncional en los planos local, nacional e incluso global. Los antropólogos han observado que, por lo general, las personas tienen dos maneras para lidiar con los significados de la comida étnica/marginal: para resaltar o para ocultar los vínculos de afiliación étnica de la comida. La primera se ha utilizado principalmente con fines políticos, tales como el establecimiento de una identidad o la creación de una conexión con ciertos grupos étnicos mediante el consumo de sus alimentos característicos o representativos (por ejemplo, sinonggi, la harina de sagú en Indonesia). ${ }^{7}$ La segunda se ha producido a raíz de la comercialización de los alimentos étnicos entre los consumidores en general; como lo demuestran los estudios de caso sobre el aguacate en Estados Unidos, ${ }^{8}$ la carne de alpaca en Perú, ${ }^{9}$ y los mijos menores en India. ${ }^{10}$ Este estudio aporta un caso en el que la comida local en las zonas de las minorías étnicas se comercializa en conjunto con sus afiliaciones étnicas.

Los estudios han demostrado que los significados simbólicos relacionados con la comida étnica tienen importancia y que, como dijo Finnis, "el éxito de un intento de llevar un alimento marginal hacia contextos más amplios puede depender de que su estatus simbólico sea reposicionado de manera eficiente". ${ }^{11}$ Ocultar las asociaciones étnicas de los alimentos ha sido una de las estrategias clave utilizadas en dicho reposicionamiento, como en los casos del aguacate, del mijo menor y la carne de alpaca en Estados Unidos, India y Perú respectivamente.

${ }^{6}$ Véase Finnis (ed.), Reimagining Marginalized Foods, op. cit.

${ }^{7}$ Véase Wini P. Utari, "Redefining the Cultural Meanings of Sinonggi during the Indonesian Decentralization Era”, en Finnis (ed.), Reimagining Marginalized Foods, op. cit., pp. 49-66.

${ }^{8}$ Jeffrey Charles, "Searching for Gold in Guacamole: California Growers Market the Avocado, 1910-1994", en Warren Belasco y Philip Scranton (eds.), Food Nations: Selling Taste in Consumer Societies, Nueva York, Routledge, 2002, pp. 131-155.

${ }_{9}^{9}$ Lisa Markowitz, "Highland Haute Cuisine: The Transformation of Alpaca Meat”, en Finnis (ed.), Reimagining Marginalized Foods, op. cit., pp. 34-48.

${ }^{10}$ Elizabeth Finnis, "Redefining and Re-presenting Minor Millets in South India", en Finnis (ed.), Reimagining Marginalized Foods, op. cit., pp. 109-132.

${ }^{11}$ Finnis (ed.), Reimagining Marginalized Foods, op. cit., p. 9. 
Como los estudios existentes han demostrado, hay que hacer una serie de cosas con el fin de comercializar los alimentos marginales; entre ellas, crear organizaciones, reinterpretar los alimentos o volver a imaginarlos; volver a empacarlos o estandarizar tanto los alimentos como la cocina, organizar actos rituales y promover discursos de masas repetitivos. Por ejemplo, es necesario seguir algunas de las prácticas populares actuales en la industria alimentaria y restaurantera, y reorganizar los alimentos en ciertas formas modernas (es decir, en menús fijos o en comidas con una estructura determinada) y decir más acerca de sus ventajas y valores mientras se borra su historia con el fin de sustituir sus significados negativos con significados positivos y atractivos. ${ }^{12}$ Este tipo de estandarización ayuda a los clientes a aceptar los alimentos con mayor facilidad. ${ }^{13}$ En comparación con la comercialización de los alimentos marginales en los países mencionados, la política propia, la historia y la cultura de las minorías étnicas en China han hecho que los significados y la comercialización de sus alimentos locales se compliquen aún más.

Como un país de gran tamaño, China ha diversificado sus costumbres alimentarias en diferentes áreas. La gente ha tratado de clasificarlas en tipos de cocina (caixi), y esto ha dado como resultado cinco categorías, ocho categorías o diez categorías. ${ }^{14}$ De hecho, como lo afirma Eugene Anderson y ha sido demostrado por una nueva serie de televisión muy popular en China titulada Un bocado de China, hay una gran cantidad de zonas de minorías étnicas y marginales que no han sido consideradas en estas clasificaciones. ${ }^{15}$

La comida local provee una de las ventanas esenciales para que los académicos estudien la economía, la sociedad, la cultu-

${ }^{12}$ Markowitz, "Highland Haute Cuisine”, op. cit., p. 40.

${ }^{13}$ Josephine Smart, "Ethnic Entrepreneurship, Transmigration, and Social Integration: An Ethnographic Study of Chinese Restaurant Owners in Rural Western Canada", Urban Anthropology and Studies of Cultural Systems and World Economic Development, vol. 32, núm. 3-4, 2003, pp. 311-342.

${ }^{14}$ No se trata de clasificaciones formales sino que sólo existen en el discurso público. El grupo de cinco categorías incluye las cocinas Guangdong, Sichuan, Hunan, Shandong y Fujian; al de ocho se suman las cocinas Jiangsu, Zhejiang y Anhui; y al de diez, las cocinas Hubei y Beijing. 1988.

${ }^{15}$ Eugene N. Anderson, The Food of China, New Haven, Yale University Press, 
ra y la política de la China contemporánea. Aproximadamente desde finales de 1980, la comida local en China ha sido reinventada y reempaquetada para los consumidores urbanos e incluso para los extranjeros, y ha comenzado a ingresar en los sistemas de restaurantes urbanos con la etiqueta de "platillos locales" (benbi cai), "comida del diario" (jiachang cai), "comida de agricultores" (nongjia cai) o "comida étnica" (minzufengrei). ${ }^{16} \mathrm{Un}$ ejemplo notable es el del condado de Shaxian, en la provincia de Fujian, al sureste de China, que ha comercializado con éxito su comida local en muchas ciudades del país, incluso en las áreas de comida de muchos campus universitarios; es más, en las últimas dos décadas, un proyecto de desarrollo turístico rural para construir restaurantes llamados "Felicidad del agricultor" (nongjiale) ha sido apoyado y regulado por la Oficina de Turismo de China y ha llegado a casi todos los condados del país. Los restaurantes "Felicidad del agricultor" ayudan a integrar las culturas alimentarias locales con el desarrollo de la industria del turismo. En contraste con la fama de dichos restaurantes o de la comida regional (es decir, de la comida local de Shaxian, los fideos Lanzhou o los fideos de arroz Guilin), sólo unos pocos de mis entrevistados conocen un restaurante en China que lleve el nombre de un grupo étnico.

Este artículo se centra en la comida local y sus significados asociados en la zona étnica de China central, y se basa en las investigaciones de campo realizadas en la prefectura de Enshi, una prefectura autónoma Tujia-Miao que se encuentra en el suroeste de la provincia de Hubei. Enshi se conoce como "la prefectura autónoma étnica más joven del país” porque apenas fue establecida en diciembre de 1983 (antes de 1983 fue tratada como una zona no étnica). Está conformada por ocho condados, incluidos Badong, Hefeng, Jianshi, Laifeng, Xianfeng, Xuanen y Enshi, y tiene una superficie total de 23963 kilómetros cuadrados y una población de más de 3.8 millones de habitantes. Esta prefectura es famosa por sus montañas, agri-

${ }^{16}$ También los restaurantes "Comiendo amargura" (yiku fan) que surgieron en la década de 1990 con temas sobre la Revolución Cultural; véase Michael Griffiths, "Eating Bitterness: Re-enacting the Primitive Rural", en L. Hernandez y S. Krajewski (eds.), Crossing Cultural Boundaries: Taboos, Bodies and Identities, Cambridge, Cambridge University Press, 2009, pp. 159-173. 
cultura y herencias étnicas. Es mayoritariamente montañosa: la montaña Wuling se encuentra en el sur, la montaña Wushan en el norte, la montaña Dalou en el oeste, y la montaña Daba en el noroeste. El cultivo intensivo del maíz, la papa, el arroz y otros cultivos de montaña a gran escala no comenzó sino hasta mediados del siglo XVIII. Hasta la fecha, excepto por el turismo y el té, la prefectura de Enshi tiene pocas industrias y sigue siendo una zona típicamente agrícola. De acuerdo con las publicaciones oficiales locales, más de diez grupos étnicos (min$z u)$ viven en esta prefectura, ${ }^{17}$ entre los cuales los más grandes son los han, tujia, miao y dong. Este patrón multiétnico fue resultado de los movimientos de "identificación étnica" de las décadas de 1950 y 1980, que se llevaron a cabo mediante "la aplicación flexible de la teoría de Stalin sobre la etnicidad". ${ }^{18}$ A través de los movimientos de identificación étnica y la creación de la prefectura étnica, la marginalidad de Enshi ha sido plenamente establecida: es una zona montañosa, de minorías, de pobreza y de tierras remotas.

Al igual que en muchas otras zonas de la China contemporánea, la comida local en Enshi, conocida como comida bezha, en las últimas décadas ha sido integrada en el sistema de restaurantes urbanos y ha sido asociada por los medios de comunicación locales y los discursos de masas con distintos significados; como comida del diario, comida de agricultores, comida de pobres, comida de montaña, comida de hambruna, comida étnica, comida sana y sabrosa, manjares en las mesas de los extranjeros y otros por el estilo. A principios de 1990, cuando trabajaba para el gobierno de la prefectura de Enshi, surgió un gran número de restaurantes especializados en la venta de comida local en Enshi que atrajo la atención tanto de los funcionarios gubernamentales como de los citadinos comunes y generó una serie de discursos locales de masas sobre la comida local. Muchos de mis colegas en el gobierno creían que estos restaurantes existían sólo para los migrantes rurales que hacían

${ }^{17}$ Enshi Zhouminwei (Comité de Asuntos Étnicos de la Prefectura Enshi), Exi Zizhizhou minzu zhi (Crónica étnica de la prefectura autónoma Exi), Chengdu, Sichuan minzu chubanshe, 1993, pp. 10, 15.

${ }^{18}$ Thomas Heberer, China and Its National Minorities: Autonomy or Assimilation?, Nueva York, M. E. Sharpe, 1989. 
trabajos manuales en las ciudades, ya que sólo la comida local era barata y podía mantener a la gente llena de energía. Entre los años 2000 y 2001, dirigí un trabajo de campo antropológico en Enshi que estaba centrado en la comercialización y en los significados de esta comida local. En la prefectura de Enshi, varios platillos locales especiales, como bezha, baogufan (un platillo hecho de harina de maíz y arroz) y zhaguangjiao (una conserva hecha de harina de maíz y chile rojo), por mucho tiempo habían sido considerados como representativos de la comida regional local o de la cocina campesina "rústica". En el periodo republicano (1911-1949), cuando Enshi no se consideraba como un "área étnica", estos alimentos se etiquetaron oficialmente como símbolos de "la comida de las zonas montañosas” y, más tarde, cuando Enshi se convirtió en zona "étnica”, fueron considerados como "comida tujia". ${ }^{19}$

Mediante el análisis de la comida local en Enshi, este artículo describe la historia de la comida hezha y examina cómo y por qué la comida local ha sido integrada en estos restaurantes y ha sido estandarizada por ellos; también analiza las razones que explican la coexistencia de una gran cantidad de significados contradictorios que a menudo provocan mucha confusión entre los observadores y los consumidores.

\section{La comida hezha y el Estado en el suroeste de Hubei}

A principios del siglo XVIII, no existía en Enshi la comida local (representada por platillos de maíz y soja) conocida como comida bezha. ${ }^{20} \mathrm{~A}$ pesar de que el maíz era considerado un gran cultivo que ayudaba a la cultura de subversión en otros lugares como Zomia, aproximadamente sólo hasta mediados del siglo XVIII, cuando se introdujo el maíz, los alimentos hezha comenzaron

${ }^{19}$ Véase Enshi Zhouminwei (Comité de Asuntos Étnicos de la Prefectura de Enshi), Exi Zizhizhou gaikuang (Resumen de la prefectura autónoma de Exi Tujia y Miao), Wuhan, Hubei renmin chubanshe, 1990, p. 25; Xiusong Deng et al., "Kan 'Hezha' zengyang daihe yifang jingji”' (Cómo ha impulsado hezha la economía regional), Hubei ribao, 12 de noviembre de 1997, p. 1. Además, un restaurante Hezha afirma que cuenta con la insuperable comida tujia.

${ }^{20} \mathrm{Gu}$ Cai (Orig. 1704), Rongmei jiyou (Los viajes de Rongmei), comentado por Gao Runsheng, Tianjing, Tianjing guji chubanshe, 1991. 
a desempeñar un papel esencial para ayudar al gobierno central a establecer un control directo de las zonas montañosas en el suroeste de Hubei. ${ }^{21}$ En la historia, el Estado, para fines de control, prefería ciertos cultivos de alimentos y rechazaba otros. Algunos cereales, especialmente el arroz, eran sus favoritos. El cultivo del arroz fue capaz de propiciar la "densidad en la concentración de la población y del grano" en las llanuras, donde por lo general se han ubicado los estados en el pasado. ${ }^{22}$ El maíz, sin embargo, fue el cultivo de montaña perfecto; tenía un alto rendimiento y podía ayudar a las personas a evadir al Estado y a sobrevivir en áreas que "eran demasiado altas, demasiado inclinadas, demasiado secas y demasiado infértiles". ${ }^{23}$

Hasta 1735, el suroeste de Hubei fue una región que siempre había estado controlada por jefes locales. Fue en ese año cuando el emperador Yongzheng de la dinastía Qing (16441911) finalmente reemplazó a estos jefes con funcionarios designados por el gobierno Qing, $y$ desde entonces la región estuvo bajo el régimen directo del gobierno central. En el siglo XVIII, las nuevas autoridades hicieron todo lo posible para reclutar migrantes de fuera y atraerlos hacia esta zona de montaña. Llegaron muchos inmigrantes de las llanuras de Yangtze y de las llamadas áreas zomia en el suroeste de Chin $\mathrm{a}^{24}$ que introdujeron en Enshi muchos nuevos cultivos, incluidos el maíz, la papa, el camote, el chile y nuevas variedades de arroz. En poco tiempo se estableció un nuevo sistema alimentario a base de maíz y soja, junto con otros cultivos secundarios y plantas silvestres comestibles. Este sistema alimentario ha apoyado también el desarrollo de una nueva identidad regional en Enshi, comúnmente compartida por los habitantes de las montañas. ${ }^{25}$

Debido al hecho de que la población local ha dependido en gran medida del maíz en lugar del arroz, por no hablar de la recolección regular de plantas silvestres comestibles en ciertas

${ }^{21}$ Scott, The Art of not being governed, op. cit.

${ }^{22}$ Ibid., p. 74.

${ }^{23}$ Ibid., p. 201.

${ }^{24} \mathrm{Idem}$.

${ }^{25} \mathrm{Xu}$ Wu, Farming, Cooking, and Eating Practices in the Central China Highland: How Hezha Foods function to establish Ethnic Identity, Lewiston, Edwin Mellen Press, 2011. 
épocas del año, desde el siglo XVIII, en la perspectiva administrativa, las autoridades locales han desarrollado una mentalidad de preocupación por el hambre; específicamente, de preparación para sortear el hambre y las hambrunas. Las autoridades trataron de promover el cultivo del arroz, que era el símbolo de la vida civilizada, ya que la historia local registraba que "todas las áreas planas en las montañas estaban siendo convertidas en arrozales". ${ }^{26}$ Hasta principios de la década de 1990, el platillo habitual hecho de maíz aún servía como indicador de la vida miserable de la montaña. A principios de 1990, cuando el gobierno chino puso en marcha un proyecto de desarrollo rural en todo el país, los funcionarios de Enshi fueron a las cocinas de los aldeanos para comprobar si el plato de maíz había sido reemplazado por el de arroz.

Desde la perspectiva nutricional, los platillos que combinan el maíz y la soja pueden proporcionar nutrientes bien balanceados y suficiente energía para el cuerpo humano. Con el alto y confiable rendimiento del maíz en los campos montañosos y la sabiduría culinaria de la población local, los alimentos bezha han jugado un papel esencial en la historia moderna de esta zona montañosa.

\section{Los restaurantes Hezha}

Antes de ser utilizado como el nombre de una marca de restaurantes, hezha era el nombre de un platillo local común: una sopa con soja líquida (con restos de frijol) y verduras picadas como ingrediente principal. Este platillo bezha es tan común que ha sido utilizado como metáfora de la comida del diario, y en las conversaciones cotidianas de la gente, "hacer bezha" significa simplemente preparar una comida. Por ello, es comprensible que, a finales de 1980, cuando una mujer rural que vivía en la zona fronteriza entre Xuanen y los condados Xianfeng decidió comercializar la comida local en su restaurante, optara por llamarlo restaurante Hezha. 
El platillo hezha suele consumirse junto con otro platillo local, baogufan, un plato principal hecho de harina de maíz mezclada con arroz (antes de la década de 1980, muchos de los pobladores de esta zona comían el platillo principal sólo con harina de maíz, que era considerada un símbolo de la vida pobre). Esta estructura del "platillo hezha y harina de maíz" se ha convertido en representativa de la vida en la montaña y de la pobreza. Por eso, en el desarrollo rural de principios de la década de 1990, sustituir la harina de maíz con un plato de puro arroz era indicativo de la mejora en el nivel de vida de los pobladores.

Sin embargo, con el desarrollo rural, los alimentos de los pobres no desaparecieron del todo; por el contrario, incluso encontraron la manera de llegar hasta el sistema urbano de restaurantes. El trabajo de campo realizado en varias aldeas, durante 2000-2001, demostró que la mayoría de los agricultores locales estaba convencido de que disfrutaba de un mejor nivel de vida que en la década de 1970. Por ejemplo, muchos dijeron que tenían acceso, más fácil que nunca, a la carne y al arroz (distintivos de un mejor nivel de vida y, a menudo, asociados con el grupo étnico han, la mayoría). La mayor parte de los agricultores atribuyeron estas mejoras a la agricultura científica iniciada en la década de 1970 y a la reforma agraria de la década de 1980 que asignó a cada hogar tierras colectivas a través de contratos. Sin embargo, se ha hecho evidente otra tendencia en las costumbres alimentarias locales. Desde mediados de la década de 1990, la prefectura de Enshi ha sido testigo de un fenómeno notorio entre la población local: el regreso a los alimentos locales "rústicos" a pesar de la abundancia de carne y arroz. En los medios de comunicación local, esta tendencia se conoce como "volver a lo original". ${ }^{27}$ Los ejemplos más representativos son: $i$ ) el uso común de la harina de maíz en lugar del arroz en los platillos principales de la población local, a pesar de que se pensaba que era inferior al arroz (fan), y ii) el crecimiento inesperado de los restaurantes Hezha, que se especializan en la venta de comida local. El platillo de soja (hezha) y los platillos principales especiales y locales ( $\mathrm{fan}$ ) como el baogufan, una hierba silvestre (Hout-

${ }^{27}$ Xiusong Deng et al., "Kan 'Hezha' zengyang daihe yifang jingji”, op. cit., p. 1. 
tuynia cordata) llamada "hierba con olor a pescado" (yuxing cao) y el almidón de helecho silvestre (generalmente etiquetado como alimento de hambruna), todos encontraron su camino para ser incorporados en los restaurantes urbanos. Es más, la Houttuynia cordata, también llamada raíz con orejas de lado (ceer gen), se utiliza incluso como uno de los platillos fríos en las mesas de los banquetes formales locales.

La señora que ayudó a crear un nicho para los alimentos hezha en el sistema de restaurantes comentó que en 1980 había abierto su primer restaurante Hezha en su pueblo, Zhang, ubicado al lado de la carretera que conecta a Enshi con la región de Xianfeng. En ese momento, ella sólo deseaba ganarse la vida vendiéndoles comida local a los choferes y los pasajeros (la mayoría de los cuales eran trabajadores rurales migrantes) que pasaban por el camino, y no tenía ambición alguna de hacer mucho dinero. Sin embargo, desde la década de 1990, inesperadamente su restaurante se volvió famoso y más y más clientes se sintieron atraídos a esta remota aldea sólo para comer la comida hezha. Para el año 2000, además de las tres sucursales que ya habían abierto en su pueblo natal, que para entonces era una pequeña ciudad, sus hijos tenían otras cinco sucursales en diferentes ciudades y pueblos. En 2011, descubrí que la sucursal ubicada en la ciudad de Enshi se había convertido en un restaurante de lujo.

El surgimiento y la expansión de los restaurantes Hezha representan un aspecto de los cambios sociales drásticos que se dieron durante la era de la Reforma en China: las tendencias de la migración del campo a las ciudades y los suburbios de las grandes ciudades y el crecimiento de una población flotante después de la aplicación del sistema de responsabilidad en la China rural. ${ }^{28} \mathrm{~A}$ menudo, la comida seguía a las personas. El surgimiento y la expansión de los restaurantes Hezha reflejan ese carácter de la época.

En poco tiempo, los clientes de los restaurantes Hezha aumentaron. Los primeros comensales regulares fueron los migrantes rurales y los choferes; luego siguieron los habitantes de

${ }^{28}$ Hein Mallee, "Migration, Hukou and Resistance in Reform China", en Elizabeth Perry y Mark Selden (eds.), Chinese Society: Change, Conflict and Resistance, Londres-Nueva York, Routledge, 2000, pp. 83-101. 
la ciudad, los funcionarios y finalmente la gente que provenía de otras prefecturas e incluso de otras provincias. A principios de 2001, cuando se estaba reconstruyendo una carretera en Enshi, el tráfico en ese camino se desvió a través del pueblo de Zhang. Este desvío, sin embargo, no molestó ni a los conductores ni a los pasajeros, ya que tuvieron la oportunidad de comer en el primer restaurante Hezha. En aquellos días, cuando estaba realizando mi investigación, el dueño del restaurante a menudo contaba sonriente el número de automóviles y camiones provenientes de otras provincias que se habían detenido en el patio de su restaurante. Durante la primera década del año 2000, de acuerdo con el propietario de un restaurante Hezha en el pueblo de Zhang, los clientes más habituales de su restaurante eran funcionarios locales, tanto del poblado de Xiaoguan como de la ciudad de Xianfeng, quienes en repetidas ocasiones se habían trasladado hasta allí para disfrutar una comida hezha. Por ejemplo, cuando los altos funcionarios llegaban de fuera, por lo general los cuadros locales los atendían en este restaurante ofreciéndoles platillos hezha dos veces: una durante el primer encuentro y la otra para despedirlos. Influidos por el entusiasmo de la gente de Enshi por los restaurantes Hezha, muchos de esos nuevos clientes comenzaron a probar esta comida de la montaña. Cuando los forasteros apenas estaban empezando a aceptar y desarrollar su gusto por la comida local, muchas personas en Enshi ya eran capaces de percibir pequeñas diferencias entre los platillos de soja líquida de los diferentes restaurantes Hezha. Por ejemplo, un joven que había comido el platillo en la aldea de Zhang y luego fue a la sucursal de Enshi, me dijo que entre los dos platillos hezha había pequeñas diferencias.

\section{La estandarización en los restaurantes Hezha ${ }^{29}$}

Mary Douglas ${ }^{30}$ sostiene que cada comida se construye de acuerdo con ciertas reglas, las cuales trazan sus raíces sociales y culturales. Para reempacar la comida local para los consumidores

${ }^{29}$ Los materiales de esta sección fueron publicados originalmente en uno de mis artículos anteriores (véase Xu Wu, “Ethnic Foods' and Regional Identity: The Hezha Restaurants in Enshi”, Food and Foodways, vol. 12, núm. 4, 2004, pp. 225-246).

${ }^{30}$ Mary Douglas, "Deciphering a Meal”, Daedalus, vol. 101, 1972, pp. 61-81. 
urbanos, los creadores de los restaurantes Hezha combinaron la nueva práctica de un menú fijo, introducida por la comida rápida occidental, con la estructura estándar de la comida china de los platillos fan y cai, e identificaron los platillos representativos de la comida local en Enshi. Una comparación de la comida de los restaurantes Hezha con la comida del diario de los lugareños indica, sin excepción, que todos los platillos de los restaurantes Hezha no se construyeron al azar, sino que se basan por completo en las costumbres alimentarias populares locales: los ingredientes, el olor, el sabor y los colores de los platillos se derivan enteramente de las prácticas tradicionales locales; es más, durante las fiestas populares locales, los restaurantes también sirven la comida especial de las fiestas.

Durante la primera década de 2000, los restaurantes Hezha no ofrecían comida a la carta ni por pedido, sino sólo un menú fijo; pero en la década de 2010 presentaron un menú a la carta y pidieron a los clientes que escogieran y ordenaran el platillo principal (el hezha y otros platillos todavía conforman un menú fijo). ${ }^{31}$ En 2000-2001, el precio de un menú fijo en un restaurante Hezha era de ocho yuanes (alrededor de un dólar estadounidense) y en 2011, de cuarenta yuanes (unos seis dólares) por persona. Los clientes podían pedir platillos hezha adicionales sin cargo extra. En estos restaurantes por lo común los platillos se sirven rápidamente. El camarero lleva a los clientes a una mesa y rápidamente les sirve dos guisos en ollas calientes, además de la bebida especial local llamada sopa de té de aceite (youcha). Uno de ellos es la sopa hezha; el otro, carne de cerdo mezclada con tofu seco. Muy pronto se sirve el resto de los platillos, quince más, todos ellos contenidos en pequeños recipientes. Los quince tiempos incluyen alimentos fríos, otros salteados o elaborados en freidoras, un plato cocido al vapor y otro de estofado. De acuerdo con la dicotomía tradicional china en la clasificación de alimentos (es decir, cai, los platillos de vegetales o de carne, y fan, los platillos principales), ${ }^{32}$ los diecisiete tiempos arriba

${ }^{31}$ Se dice que ordenar el plato principal es una práctica tomada de los restaurantes "Felicidad del agricultor".

${ }^{32}$ V'ease Kwang-Chih Chang, "Introduction", en K. C. Chang (ed.), Food in Chinese Culture: Anthropological and Historical Perspectives, New Haven, Yale University Press, 1977, pp. 3-21. 
citados (con excepción del té de aceite) pertenecen a la categoría de verdura o carne (cai). Un restaurante Hezha servía otros tres platillos regulares como plato principal, que incluían un plato de arroz blanco (baifan), un plato de maíz en polvo (baogufan) y una papa al vapor mezclada con arroz (yangyufan). En el segundo mes lunar, durante el Festival del Sacrificio de la Primavera (Sheri), este restaurante también preparaba el platillo de ajenjo (shefan), que estaba hecho de carne de cerdo y tripa ahumadas, arroz glutinoso, arroz regular y ajenjo y ajo silvestres. Incluido el shefan, todos los platillos principales se cuecen al vapor. El licor de maíz producido localmente (baogujiu) es la única bebida alcohólica que ofrece el restaurante para el consumo de sus clientes. Todas estas prácticas contribuyen a la autenticidad de los restaurantes Hezha.

Desde la perspectiva del sabor y de la textura, un informante local describió con cuatro palabras las características generales de la comida hezha: ácido, dulce, glutinoso y picante. En términos generales, los sabores ácidos y picantes se utilizan en los platillos que se elaboran para las comidas del diario, mientras que los alimentos dulces y glutinosos se preparan para los días especiales, como los de los festivales. Los platillos que conformaban los menús fijos de los restaurantes Hezha reflejaban plenamente estas características.

A la gente de Enshi le gusta mucho el sabor agrio y cree que los alimentos agrios son buenos para estimular el apetito. En los hogares de esta zona se envasan en frascos las verduras en escabeche (en vinagre) y en conserva. Cada familia posee al menos un par de tarros de diferente tamaño para hacer vegetales en vinagre (suancai o paocai, como chiles, col china, legumbres, ajo, soja, cebolla, nabo, rábano, brotes de bambú y col) y conservas vegetales (yancai, como la harina de maíz mezclada con pimiento rojo, el pimiento rojo machado en vinagre, el rábano en escabeche y el tubérculo de mostaza encurtido, zhacai).

En el pasado no había azúcar blanca refinada ni azúcar moscabado, pero la gente de Enshi tenía sus propias formas de hacer azúcar; por ejemplo, a partir del camote y del maíz. Este tipo de azúcar es muy dura, por lo que es necesario un cuchillo de hierro para cortarla. También hacen un alcohol dulce, que se conoce con el nombre de "alcohol de arroz" o laozao, poniendo 
el arroz glutinoso al vapor y fermentándolo. A algunas familias les gusta añadir un poco de maíz aplastado en el arroz glutinoso; se decía que a las personas que sufren de enfermedades estomacales les gusta especialmente este alcohol dulce hecho con maíz agregado. El alcohol dulce contiene un toque de alcohol y un rastro de dulzor; se sirve frío en verano y caliente en invierno. La práctica de la apicultura para la obtención de miel ha sido también una tradición de mucho tiempo, ${ }^{33} \mathrm{y}$ a algunos ancianos les gusta agregar miel al alcohol. El alcohol de maíz también tiene un dejo de dulzor. Por esta razón, en las reuniones la gente del lugar propone beber con la cena el alcohol del maíz producido localmente. En el menú fijo de los restaurantes Hezha hay un platillo hecho con bolas de camote.

Hay muchos tipos de comida especial para las fiestas elaborada con arroz glutinoso; por ejemplo, el pastel de arroz pegajoso, ciba (a algunas familias les gusta añadir algo de maíz aplastado al pastel ciba, que por lo general está hecho con $70 \%$ de arroz glutinoso y $30 \%$ de arroz regular); el alcohol de arroz (tianjiu); los dumplings (bolitas de masa) hechos de arroz glutinoso (tangyuan); los fideos de arroz regular y arroz glutinoso (doupi), y el platillo de ajenjo. La población local prefiere hacer el platillo de arroz glutinoso (nuomifan) en lugar del platillo principal normal que se hace de arroz que no se pega. Debido a su uso en las comidas para las fiestas, el arroz glutinoso todavía es plantado cada año por algunos agricultores, a pesar de que su rendimiento es menor al del arroz regular.

No es una exageración decir que "en Enshi ninguna familia puede dejar de comer chile en cada comida”. El chile se consume de diferentes maneras: i) como chile verde o rojo sofrito, a veces preparado con carne de cerdo ahumada; ii) como chile rojo seco y ahumado, que es uno de los condimentos; iii) como conserva de chile rojo, que puede utilizarse como condimento o como plato frío independiente; $i v$ ) como chile en vinagre, que puede consumirse como plato frío, y v) como salsa de chile, que se hace con chile rojo picado, harina y las cinco especias y que es utilizada como condimento. La Lindera glauca, una especia local silvestre, es considerada alimento picante. A menudo

${ }^{33} \mathrm{Gu}$ Cai, Rongmei jiyou, op. cit. 
se hace en escabeche con salsa o se conserva con chile rojo; su función es ayudar a regular la esencia ( $q i)$ de las personas; es decir, regula el flujo de energía vital y elimina sus obstáculos.

Además de las cuatro características de sabor y textura mencionadas antes, el maíz, la soja y las plantas silvestres comestibles están muy involucrados en el sistema alimentario bezha y simbolizan la identidad regional. En Enshi, la soja es usada en varias docenas de platillos, como el tofu y el plato local especial hezha. Me informaron que incluso antes de 1949, ya eran famosos el platillo hezha y los pastelillos de arroz hechos en el municipio de Xiaoguan, en el condado de Xuanen. Algunos dichos locales, como el de "consumir chile como sal [condimento] y hacer hezha [en lugar de carne] para celebrar el año nuevo", han sido citados siempre por la élite como prueba de lo pobres que eran las familias en las altas montañas. Sin embargo, esto tal vez puede interpretarse de otra manera: que a la población local realmente le encantaba su comida.

Al descifrar el menú del día en un restaurante Hezha, a principios de la década de 2000 , descubrí que éste respondía a la estructura "un plato principal, una guarnición y una bebida”, además de diferentes estrategias para cocinar y diferentes estrategias para la adquisición de alimentos. Una característica principal del sistema de la cocina de Enshi es que se basa en las reglas de la comida local. La gente de Enshi tiene una serie de normas alimentarias sobre la forma en la que deben mezclarse o combinarse los alimentos, sobre lo que debe incluirse en cada una de las comidas diarias, lo que debe comerse para celebrar las fiestas y los banquetes, así como una serie de ideas para las terapias basadas en dietas. A las personas oriundas de Enshi les gusta mezclar todo, y lo mismo puede decirse de los sabores en la comida. Los alimentos que combinan los sabores agrios y picantes parecen ser un rasgo distintivo de su cocina. El zhaguangjiao (conserva de harina de maíz y chile picado) es representativo de este tipo de alimentos; puede cocinarse sofrito con cerdo ahumado o puede cocerse en una pasta vertiendo sobre él un poco de agua. Los ocho condados de la prefectura de Enshi tienen este platillo. Por lo tanto, en el restaurante Hezha, el platillo bezha, el cerdo ahumado (o los sedimentos de la grasa de cerdo) frito con zhaguangjiao, las raíces de la hierba 
con olor a pescado bañadas en salsa, y la harina de maíz cocida al vapor constituyen los cuatro muy llamativos y representativos tiempos del menú fijo.

Independientemente de sus afiliaciones étnicas, la gente de Enshi por lo general hace tres comidas al día, dos de las cuales deben incluir el formato de un "plato principal y una guarnición (plato de verduras y carne)". Los platillos principales son generalmente alimentos básicos al vapor, como arroz, maíz, papa, etcétera, y los platillos cai están hechos de carne y verduras, aunque algunas veces pueden contener arroz, papa o camote. En Enshi, el trigo, a pesar de que es considerado un "cultivo principal”, no se utiliza como alimento básico, y cae tanto en la categoría de platillo cai como en la de bocadillo (lingshi). Las dicotomías más básicas en el concepto de comida para la gente de Enshi son: "platillo fan contra platillo cai" y "plato principal (zbuliang) frente a guarnición (zaliang)". Entonces, de acuerdo con la clasificación local de plato principal, platillo de vegetales y carne, y bebidas, las comidas descritas pueden considerarse una estructura completa del sistema " $f a n$-cai más bebida".

La gente de Enshi suele utilizar siete métodos para preparar los alimentos: sofreír (chao), freír en abundante aceite con una freidora (zha), estofar (dun), cocer al vapor (zheng), freír en poco aceite (jian), encurtir (yan), aderezar con salsa las verduras crudas (liangban) y estofar en olla $(l u)$. Con frecuencia, el método de cocimiento al vapor se utiliza para cocinar los platillos principales. Cuando se sofríe, el fuego debe ser lo suficientemente fuerte como para dejar que los alimentos se cocinen muy rápidamente, porque a la gente de Enshi le gusta mucho el olor de la comida frita rápidamente (chao-cai), y también pueden reconocer incluso las pequeñas diferencias entre los sabores de los platillos chao-cai cocinados en un fuego fuerte, y los de los cocinados a fuego lento. En el menú fijo del restaurante Hezha de Zhangguan hay por lo menos un guiso de cada una de las siete categorías anteriores. Además, debe existir un platillo hecho de plantas silvestres comestibles, como la hierba con olor a pescado, o las rebanadas de fécula de helechos sofritas. Por lo tanto, de acuerdo con los métodos locales de preparación de alimentos, este menú fijo puede ser visto como 
una estructura de las distintas estrategias para cocinar comúnmente utilizadas por la población local: platillos fríos, salteados, fritos, al vapor y estofados.

Ciertos alimentos son necesarios para la celebración de los festivales. Por ejemplo, los aldeanos tienen un buen número de platillos que requieren de arroz glutinoso. El vino de arroz (vino dulce, tianjiu) es un favorito enshiense. Como la gente dice: "Lo hacemos no sólo para celebrar las fiestas, sino también para los días normales, los cumpleaños y los periodos de cultivo más intensos, como los destinados a trasplantar las plántulas de arroz". Varios pasteles, entre ellos el hecho de polvo de arroz glutinoso y de polvo de arroz regular (fenba), las bolitas de arroz y el pastel de arroz (ciba), se preparan para celebrar el festival de año nuevo; por ello, la gente de la localidad necesita más arroz glutinoso en ese momento, e incluso las personas a las que no les gusta ese tipo de arroz piensan que "debe seguirse preparando para la celebración de los festivales”. Cuando la gente en Enshi va a los banquetes de la comunidad dice que va a "comer vino" (chijiu), ya que en un banquete debe ofrecerse vino y más platillos de carne que comidas regulares. Todos los banquetes se clasifican como eventos felices rojos (hongshi) y eventos felices blancos (baishi), once de los cuales muestran un formato de platillo básico. Teniendo como base el conjunto de platillos para una mesa de banquete, los banquetes pueden ser clasificados en "banquetes comunes" para los comensales habituales y banquetes para los "parientes importantes" (gaoqin $x i)$, que incluyen dos platillos de alta calidad: pierna de cerdo al vapor (pang) y rebanadas de carne de cerdo al vapor (kou). Durante la ceremonia, además del banquete formal también existe un banquete auxiliar que se prepara sólo para los ayudantes de cocina, y que no sigue reglas estrictas sobre el formato del platillo y el número de platos. El banquete auxiliar puede servir como experimento para que el jefe de cocina decida qué tan salados, ácidos, dulces y picantes deben quedar los platillos, de acuerdo con la opinión de los ayudantes. Así que, según las ocasiones para las que esté destinado el platillo, este menú fijo en el restaurante Hezha puede ser visto como una estructura de: comidas del diario, platillos para fiestas y platillos para banquetes formales. 
La gente de Enshi ha clasificado los alimentos en tres categorías, según su naturaleza: calientes (re), fríos (liang) y estimulantes $(f a)$. Los alimentos calientes son aquellos que tienen un sabor picante y los que aumentan el calor interno del cuerpo, como el chile, la pimienta negra, el jengibre, la cebolla, el ajo y la mandarina. Los alimentos fríos son los que alivian el calor interno, como la hierba con olor a pescado, la perilla morada y la naranja. Los alimentos estimulantes son excitantes, como la cuajada hecha de arum gigante de río (moyu-tofu), el huevo, el arroz glutinoso y las algas marinas; éstos deben ser evitados por personas que tienen enfermedades de la piel como el acné. La gente en la aldea de Lian considera que todos los alimentos ricos en nutrientes son alimentos estimulantes; por lo tanto, este menú fijo puede ser visto como una estructura de los tres tipos de alimentos: calientes, fríos y estimulantes.

Por último, de acuerdo con el estilo de conseguir comida, el menú fijo puede considerarse como una estructura de cereales y verduras cultivados, animales de cría y plantas silvestres recolectadas.

\section{Significado de la comida bezha}

Debido a la práctica y la historia local, así como a las influencias nacionales y mundiales, en general las comidas locales se asocian con significados cambiantes. ${ }^{34}$ Alrededor de la comida hezha, sin embargo, hay una coexistencia de significados diversificados. A lo largo de los distintos periodos históricos, la comida local en Enshi ha sido etiquetada por los medios de comunicación y los discursos de masas como: comida regular del diario, comida de agricultores, comida de pobres, comida de montaña, comida de hambruna, comida étnica, comida sana y sabrosa, manjares para las cenas de los extranjeros, y otros. En la China actual, debido a la construcción de las características étnicas y a la promoción de la modernización de la agricultura y el turismo, estos significados, positivos o negativos, han sido reconfirmados o renovados. Desde 1980, dos acontecimientos

\footnotetext{
${ }^{34}$ Finnis (ed.), Reimagining Marginalized Foods, op. cit.
} 
comenzaron a cuestionar los significados negativos: la exportación de comida local a los mercados extranjeros y el surgimiento de los restaurantes Hezha. Otros sucesos impugnaron y reconfirmaron los viejos significados negativos, que incluyen la creación de una prefectura étnica, la promoción de la agricultura científica (la modernización de la agricultura) y el desarrollo turístico.

Desde 1730, la comida local en Enshi se ha asociado con ciertos significados en diferentes periodos históricos. Entre los significados negativos que han tenido los alimentos hezha se encuentran: comida de bárbaros, comida de montaña, comida de hambruna y comida étnica. Específicamente, el epíteto de “comida de bárbaros" apareció a principios de los años inmediatamente posteriores a la reforma política de 1735 , mientras que el de "comida de pobres y de hambruna" lo hizo en el siglo XIX, y el de "comida de gente de montaña", a principios del siglo Xx (según varias crónicas locales). Estos significados estaban muy lejos de ser positivos y persistieron hasta la década de 1980, cuando la sociedad china entró en una nueva era reformista.

Después de la reforma política de 1735, al igual que Wang Xiemeng, un magistrado local en Enshi, los funcionarios ahí designados por el gobierno Qing se refirieron a la comida local con el adjetivo de bárbara. En su poema "La comida de la prefectura de Enshi”, Wang se mostraba muy sorprendido por lo poco común de su carácter y describió y comentó sobre los alimentos que la conformaban. Wang escribió que en esta zona salvaje, los habitantes de la localidad consumían alimentos poco comunes, como el maíz, el ñame, el hezha, una variedad de col china llamada xiuqiu baicai y un hongo que recolectaban en el bosque llamado yangdujun.

En la era de la República (1919-1949), los documentos gubernamentales se refirieron a la comida local como comida de montaña, y los alimentos que la componían sirvieron como símbolos de la vida pobre y miserable. A finales de 1950 y comienzos de 1960, una grave hambruna estalló en toda China y muchos pobladores de Enshi se vieron afectados por ella. Este trágico suceso reconfirmó el significado de la comida local como comida de hambruna. Muchos de los alimentos silvestres recolectados regularmente, como el ajenjo, el almidón de kud- 
zu, el almidón de helechos y la osmunda, jugaron un papel clave para salvar la vida de la gente, mientras que, irónicamente, al mismo tiempo refrendaban la relación que se establecía entre esa gente, la hambruna y el subdesarrollo. ${ }^{35}$

En comparación con estos significados negativos de larga duración, los significados positivos atribuidos a la comida local aparecieron más tarde; estos incluyen, principalmente, los de comida sabrosa y comida saludable. La idea de la comida sabrosa estaba relacionada con los inicios de la modernización de la agricultura en esta zona de montaña. Comida insípida y de alto rendimiento fueron dos de los primeros comentarios que hicieron los pobladores sobre los alimentos producidos con los métodos científicos modernos, que utilizan productos químicos y semillas híbridas. La idea sobre la comida sabrosa surgió primero entre los campesinos comunes locales, que amaban los alimentos tradicionales elaborados mediante sus prácticas culinarias, la variedad de sus cultivos y las formas en las que se cultivaban. En 1991, cuando yo trabajaba en un pueblo, escuché a los pobladores locales criticar abiertamente el mal sabor del arroz, de la carne de cerdo y de los huevos que se producían con los métodos científicos modernos. Mucha gente local desarrolló gradualmente un sistema dual para la producción de alimentos, ${ }^{36}$ uno para ellos con los métodos tradicionales y el otro para los consumidores urbanos con los nuevos métodos científicos. La población rural utiliza el prefijo tu-, como en tuhuevo, tutocino o tupapa, para distinguir los huevos, las papas o la carne de cerdo producidos localmente y mediante métodos tradicionales.

Otro acontecimiento que favoreció mucho los significados positivos de la comida local fue la emergencia inesperada de los mercados extranjeros para ciertos productos locales de Enshi, tales como el almidón de kudzu, el helecho, el almidón de helecho y la osmunda. La demanda de los mercados extranje-

${ }^{35} \mathrm{Xu}$ Wu, "Turning Waste into Things of Value: Marketing Fern, Kudzu and Osmunda in Enshi Prefecture, China”, Journal of Developing Societies, vol. 19, núm. 4, 2003, pp. 433-457.

${ }_{36} \mathrm{El}$ autor considera que los sistemas duales en la producción de alimentos contribuyen de manera importante a la crisis actual en la seguridad alimentaria de China, que se extendió por todo el país. 
ros y los calificativos como: "manjar en la mesa japonesa" o "rarezas de montaña" en los medios de comunicación locales, confirmaron aún más la idea de que la comida local estaba conformada por alimentos saludables. Durante las décadas de 1980 y 1990, los medios de comunicación locales, como el periódico West Hubei (Hubei Occidental) y la revista West Hubei Ethnic Economy (Economía Étnica de Hubei Occidental), además de educar a la gente sobre la necesidad de la economía de mercado y las ventajas de la agricultura moderna, también proporcionaron información valiosa sobre la manera en la que los alimentos locales, incluidas las plantas silvestres comestibles, estaban involucrados en la economía de mercado contemporánea. Para la población era interesante observar la forma en la que estos alimentos locales, que siempre habían sido tratados como despojos, asumían nuevas funciones que desempeñar (por ejemplo, en el intercambio de divisas). Inspirado en los discursos de masas, un erudito local pasó varios años recopilando y presentando información sobre muchos de los alimentos especiales locales y finalmente reunió todos esos reportes en un libro. ${ }^{37} \mathrm{Al}$ leer dichos artículos y reportajes en los medios de comunicación locales, uno podía fácilmente hacerse una idea sobre la coexistencia de los significados contradictorios asignados a los ingredientes de ese tipo de comida. Por ejemplo, un autor al mismo tiempo que hacía mención de los alimentos locales que habían sido enviados a los mercados externos y con los que se habían obtenido divisas, también hacía comentarios en el sentido de que se trataba de comida de hambruna, o que era comida de pobres. ${ }^{38}$ Cuando el almidón local de kudzu se comercializó con éxito en la década de 1990, un periodista local tituló su informe así: "Convertir los residuos en cosas de valor". ${ }^{39}$

Desde el año 2000, el discurso nacional sobre la seguridad alimentaria y los informes detallados acerca de los "suministros especiales” (tegong, alimentos especiales cultivados por métodos tradicionales para los altos funcionarios) le dieron a la gente más elementos para confiar en su comida local, conformada por

${ }_{37}$ Tian Yuande, Exi Techan Huicui (Colección de los productos locales especiales en West Hubei), Wuhan, Hubei kexuejishu chubanshe, 1993.

${ }^{38}$ Idem.

${ }^{39} \mathrm{Xu}$ Wu, "Turning Waste into Things of Value", op. cit. 
alimentos especialmente producidos de manera tradicional. Este discurso nacional sobre la seguridad alimentaria y los suministros especiales hizo de la comida local, como los alimentos hezha, algo considerablemente positivo.

El desarrollo del turismo en Enshi se ha basado en dos temas principales: las culturas étnicas y la vida sana de la montaña, los cuales involucran la comida local. Esta situación contribuye tanto a los significados positivos como a los significados negativos que se dan a la comida local. El hecho de que hoy en día el turismo se haya convertido en una prominente industria en esta zona montañosa, de alguna manera ha servido de apoyo a los significados positivos de los alimentos de la localidad, a través de la promoción de los restaurantes "Felicidad del agricultor".

Desde 1983, cuando se aprobó el estatus de Enshi como un área de minorías étnicas, se inició la construcción de las características étnicas locales. Desde entonces, la promoción turística en la prefectura de Enshi se ha centrado en los aspectos étnicos, que por lo general incluyen las costumbres y las culturas que puedan demostrar la "otredad" de las minorías y, sobre todo, su atraso. Como observaban los antropólogos: hay un principio básico, un dualismo, para la construcción étnica: la mayoría es civilizada, mientras que las minorías son primitivas. ${ }^{40}$ Tanto la comida como los otros símbolos clave, como los trajes, los rituales, los cantos y las danzas locales, son algunas de las necesidades que demuestran la alteridad. ${ }^{41}$ Los platillos locales, como el plato de maíz, el hezha y la sopa de té de aceite, se utilizan como símbolos de la otredad de las minorías locales. Posteriormente, los alimentos hezha se convirtieron en comida étnica debido a que algunos eruditos daban por hecho que existía una cultura tujia (también miao y dong) que incluye los alimentos hezha. Al convertirse en étnicos, en la década de 1980, se volvió a confirmar la idea inicial de que la comida local estaba integrada por alimentos bárbaros. El auge del turismo en la primera década del 2000 confirmó de nuevo las ca-

${ }^{40}$ Dru C. Gladney, "Representing Nationality in China: Refiguring Majority/ minority Identity", The Journal of Asian Studies, vol. 53, núm. 1, 1994, pp. 92-123.

${ }^{41}$ Susan D. Blum, Portraits of "Primitives": Ordering Human Kinds in the Chinese Nation, Nueva York, Rowman \& Littlefield Publishers, 2001. 
racterísticas étnicas (la marginalidad) de esta prefectura, y a su vez ayudó a mantener los significados negativos asociados con la comida local.

Desde la década de 1980, aproximadamente, se introdujo en China un nuevo tipo de turismo, conocido como turismo de recreo (o de vuelta a la vida tradicional saludable), que surgió en las provincias de Sichuan y Zhejiang. El consumo de los alimentos de los agricultores se convirtió en una de las principales actividades de este tipo de turismo y los lugares para comerlos se denominaron restaurantes "Felicidad del agricultor". Los aldeanos operan dichos restaurantes y pueden solicitar apoyo financiero de los gobiernos locales. Las agencias de turismo en China tienen fondos especiales y ciertas reglas para alentar a las familias a administrar los restaurantes. Desde mediados de la década de 1990, la prefectura de Enshi ha comenzado la práctica de alentar el manejo de los restaurantes "Felicidad del agricultor". Este programa turístico aumenta en gran medida el consumo de la comida local y ayuda a la difusión de sus significados positivos.

\section{Reflexiones finales}

Durante los últimos tres siglos, la gente de Enshi ha desarrollado un sistema alimentario local que los ha ayudado a adaptarse de una manera muy efectiva al entorno de la montaña, en el cual consumen la suficiente cantidad de nutrientes y con el que han logrado sustentabilidad. Basada en los alimentos hezha, la reforma política de $1730^{42}$ tuvo éxito en establecer en esta zona montañosa un sistema de gobierno centralizado. En su calidad de sistema de alimentación de montaña bien desarrollado y debido a su importancia y multifuncionalidad, la comida bezha ha recibido mucha atención de los medios de comunicación y en los discursos de masas. Desde 1730 ha tenido diversos significados.

${ }^{42}$ Hubo intentos por establecer un control directo en estas zonas de la montaña antes de la década de 1730 , pero todos fracasaron. De acuerdo con la teoría de James Scott (The Art of not being governed, op. cit.), los cultivos y el sistema alimentario podrían ser una de las principales razones de estos fracasos. 
Muy probablemente, continuarán coexistiendo los sentidos contradictorios atribuidos a la comida hezha, ya que esa coexistencia, en particular el poder de los significados negativos, proviene de la tradición del Estado de preocuparse por la cultura de evasión de su control que caracteriza a las zonas de la montaña, como quedó demostrado con la promoción de la modernización de la agricultura ${ }^{43}$ y el turismo étnico, que ha sido reforzado en parte por los aspectos étnicos que apuntan a una historia de barbarie y retraso. Debido que en China las diferencias entre las minorías étnicas y la mayoría han se interpretan oficialmente en términos de la teoría marxista sobre las diferentes etapas de la evolución social, esta construcción de las "características étnicas" de Enshi fortalece continuamente el primitivismo y la marginalidad de la población local y, en consecuencia, de sus alimentos. El poder para apoyar los significados positivos proviene de acontecimientos recientes: la lealtad de la población local a las costumbres alimentarias tradicionales, la exportación de los alimentos locales a los mercados extranjeros, el turismo de vida saludable, los conceptos y las discusiones sobre los alimentos verdes y el patrimonio agrícola $\mathrm{y}$, en particular, los llamados "suministros especiales" (tegong) para los altos funcionarios.

\section{Traducción del inglés: Carmen Arriola}

Dirección institucional del autor:

School of Social Development

East China Normal University

500 Dongchuan Road

Shanghai, 200241, PRC

xuwu919@hotmail.com

\section{Bibliografía}

Anderson, Eugene N., The Food of China, New Haven, Yale University Press, 1988.

${ }^{43} \mathrm{Scott}$, The Art of not being governed, op. cit. 
Balick, Michael J. y Paul A. Cox, Plants, People and Culture, Nueva York, Scientific American Library, 1996.

Bergman, Roland, "Subsistence Agriculture in Latin America", en John Super y Thomas Wright (eds.), Food, Politics and Society in Latin America, Lincoln, University of Nebraska Press, 1985, pp. 106-132.

Blum, Susan D., Portraits of "Primitives": Ordering Human Kinds in the Chinese Nation, Nueva York, Rowman \& Littlefield Publishers, 2001.

Chang, Kwang-Chih, "Introduction”, en K. C. Chang (ed.), Food in Chinese Culture: Anthropological and Historical Perspectives, New Haven, Yale University Press, 1977, pp. 3-21.

Charles, Jeffrey, "Searching for Gold in Guacamole: California Growers Market the Avocado, 1910-1994”, en Warren Belasco y Philip Scranton (eds.), Food Nations: Selling Taste in Consumer Societies, Nueva York, Routledge, 2002, pp. 131-155.

Deng, Xiusong et al., "Kan 'Hezha' zengyang daihe yifang jingji”, Hubei ribao, 12 de noviembre de 1997, p. 1.

Douglas, Mary, "Deciphering a Meal", Daedalus, vol. 101, 1972, pp. 61-81.

ENSHI, Zhouminwei, Exi zizhizhou minzu zhi, Chengdu, Sichuan minzu chubanshe, 1993.

ENSHI, Zhouminwei, Exi zizhizhou gaikuang, Wuhan, Hubei renmin chubanshe, 1990.

Etkin, Nina (ed.), Eating on the Wild Side: The Pharmacological, Ecological, and Social Implications of using Non-cultigens, Tucson, University of Arizona Press, 1994.

Etkin, Nina, Foods of Association, Tucson, University of Arizona Press, 2009.

FINNIS, Elizabeth (ed.), Reimagining Marginalized Foods: Global Processes, Local Places, Tucson, University of Arizona Press, 2012.

FINNIS, Elizabeth, "Redefining and Re-presenting Minor Millets in South India", en Elizabeth Finnis (ed.), Reimagining Marginalized Foods: Global Processes, Local Places, Tucson, University of Arizona Press, 2012, pp. 109-132.

FreEMAN, Milton, "Why Mattak and Other Kalaalimerngit [local foods] Matter", en Birgitte Jacobsen (ed.), Cultural and Social Research in Greenland, 95/96: Essays in Honour of Robert Petersen, Nuuk, Ilisimatusarfik-Atuakkiorfik, 1996, pp. 45-53.

Gladney, Dru C., "Representing Nationality in China: Refiguring Majority/minority Identity", The Journal of Asian Studies, vol. 53, núm. 1, 1994, pp. 92-123. 
Griffiths, Michael, "Eating Bitterness: Re-enacting the Primitive Rural”, en L. Hernandez y S. Krajewski (eds.), Crossing Cultural Boundaries: Taboos, Bodies and Identities, Cambridge, Cambridge University Press, 2009, pp. 159-173.

Gu CAI (Orig. 1704), Rongmei jiyou (The Travels in Rongmei), comentado por Gao Runsheng, Tianjing, Tianjing guji chubanshe, 1991.

Heberer, Thomas, China and Its National Minorities: Autonomy or Assimilation?, Nueva York, M. E. Sharpe, 1989.

Howard, Patricia L. (ed.), Women and Plants: Gender Relations in Biodiversity Management and Conservation, Londres-Nueva York, Zed Books, 2003.

LaIfeng XIAN, Laifeng xianzhi, Tongzhi Period (1862-1875), 1867.

MalleE, Hein, "Migration, Hukou and Resistance in Reform China", en Elizabeth Perry y Mark Selden (eds.), Chinese Society: Change, Conflict and Resistance, Londres-Nueva York, Routledge, 2000, pp. 83-101.

MarKowITZ, Lisa, "Highland Haute Cuisine: The Transformation of Alpaca Meat", en Elizabeth Finnis (ed.), Reimagining Marginalized Foods: Global Processes, Local Places, Tucson, University of Arizona Press, 2012, pp. 34-48.

NabHan, Gary Paul (ed.), Renewing America's Food Traditions: Saving and Savoring the Continent's most Endangered Foods, White River Junction, Chelsea Green Publishing, 2008.

NabHan, Gary Paul, Coming Home to Eat: The Pleasures and Politics of Local Foods, Tucson, University of Arizona Press, 2001.

NABHAN, Gary Paul, Where our Food comes From, Washington, Island Press, 2009.

Pardo-de-Santayana, Manuel, Andrea Pieroni y Rajindra K. Puri (eds.), Ethnobotany in the New Europe: People, Health and Wild Plant Resources, Nueva York, Berghahn, 2010.

Scотт, James, The Art of not being governed: Anarchist History of Upland Southeast Asia, New Haven-Londres, Yale University Press, 2009.

SMART, Josephine, "Ethnic Entrepreneurship, Transmigration, and Social Integration: An Ethnographic Study of Chinese Restaurant Owners in Rural Western Canada", Urban Anthropology and Studies of Cultural Systems and World Economic Development, vol. 32, núm. 3-4, 2003, pp. 311-342.

Tian Yuande, Exi Techan Huicui, Wuhan, Hubei kexuejishu chubanshe, 1993.

UTARI, Wini P., "Redefining the Cultural Meanings of Sinonggi during the Indonesian Decentralization Era”, en Elizabeth Finnis 
(ed.), Reimagining Marginalized Foods: Global Processes, Local Places, Tucson, University of Arizona Press, 2012, pp. 49-66.

WILK, Richard, "'Real Belizean Food': Building Local Identity in the Transnational Caribbean”, American Antbropologist, vol. 101, núm. 2, 1999, pp. 244-255.

Wu, Xu, “'Ethnic Foods' and Regional Identity: The Hezha Restaurants in Enshi", Food and Foodways, vol. 12, núm. 4, 2004, pp. 225-246.

Wu, Xu, "Turning Waste into Things of Value: Marketing Fern, Kudzu and Osmunda in Enshi Prefecture, China”, Journal of Developing Societies, vol. 19, núm. 4, 2003, pp. 433-457.

Wu, Xu, Farming, Cooking, and Eating Practices in the Central China Highland: How Hezha Foods function to establish Ethnic Identity, Lewiston, Edwin Mellen Press, 2011. 
